\title{
Two-layer modal logics: from fuzzy logics to a general framework
}

\author{
Petr Cintula ${ }^{1}$ and Carles Noguera ${ }^{12 *}$ \\ ${ }^{1}$ Institute of Computer Science, Academy of Sciences of the Czech Republic, cintula@cs.cas.cz \\ 2 Institute of Information Theory and Automation, Academy of Sciences of the Czech Republic, \\ noguera@utia.cas.cz
}

Mathematical Fuzzy Logic (MFL) started as the study of logics based on left-continuous t-norms ${ }^{1}$ most prominently Łukasiewicz logic $屯$, Gödel-Dummett logic G, Product logic П, Hájek logic BL, and the system MTL introduced by Esteva and Godo. In the last years, the scope of MFL has been progressively expanded by considering weaker logical systems characterized by their completeness with respect to a semantics of linearly algebras (such as chains endowed with a uninorm, or a non-commutative tnorm to interpret \&, or other kinds of linearly ordered residuated lattices).

There have been some proposals for a general framework to deal in a uniform way with this growing family of logics. The first one appeared in [12] when Hájek and Cintula introduced that classes of core and $\Delta$-core fuzzy logics, understood as axiomatic expansions of MTL and $\mathrm{MTL}_{\Delta}$, resp. A wider framework, encompassing weaker systems and stronger expansions, has been proposed in [3] based on the notion of weakly implicative semilinear logic. In both approaches MFL retains what we can arguably see as its defining feature, namely the study of systems of non-classical logics with a semantics based on (linearly ordered) scales of degrees of truth. This is what makes these logics specially suited for the study of gradual aspects of vagueness and imprecision, found in sentences like 'it is heavily raining' or 'that man is tall'.

A conceptually different issue, that of uncertainty, has also been addressed inside MFL. The main idea, introduced in [13] and later developed by Hájek in his monograph [11], is that one could use probability to determine the truth degree of statements such as 'tomorrow it will probably rain' or 'the probability that tomorrow it will rain is high'. Indeed, one takes classical logic and its formulae $\varphi$ to describe crisp events, introduces a new modal operator $P$ which can be applied on them to create atomic modal formulae $P \varphi$ which may be read as 'probably $\varphi$ ' (or better 'the probability of $\varphi$ is high'), and finally these atomic modal formulae are combined by using the connectives of Łukasiewicz logic. What we obtain is a two-layer modal fuzzy logic built on atomic formulae $P \varphi$ whose truth values are given by a probability measure. Several works have followed this idea with variations. In [9] Godo, Esteva and Hájek replaced Łukasiewicz logic on the second layer by ŁП, but kept classical logic for non-modal formulae. The logic $€ \Pi$, with its expanded language, enabled them to deal with conditional probability. Flaminio and Montagna also considered conditional probability in [7], and Godo and Marchioni investigated coherent conditional probabilities in [10]. Marchioni also proposed a class of logics of uncertainty in [14] with different kinds of measures (besides probability) to quantify the uncertainty of events. In all of these works classical logic has been kept as the underlying logic for non-modal formulae.

However, if one wants to deal with uncertainty and vagueness at once, i.e. with the probability of vague events, as in 'tomorrow it will probably rain heavily', the two-layer paradigm can still be useful provided that the underlying classical logic is substituted by a fuzzy logic. This idea has been also investigated in some works, as [5] where finite Łukasiewicz systems $\biguplus_{n}$ are taken as the logics of vague events. Other recent works along these lines are surveyed in [6]. There has even been a first attempt at an abstract theory of two-layer modal fuzzy logics in [15]; but it is restricted to the family of core fuzzy logics which, as argued above, has become too narrow to contain the current scope of MFL.

\footnotetext{
${ }^{*}$ The authors were supported by the Czech Science Foundation project P202/10/1826.

${ }^{1}$ For a more detailed historical account see [1].
} 
The primary aim of this paper is to provide a new general framework for two-layer modal fuzzy logics that encompasses the current state of the art and paves the way for future development. Actually, we will obtain much more than this. Indeed, we show how one can construct a modal logic (for an arbitrary modality, not necessarily read as a probability) over an arbitrary non-classical logic (under certain technical requirements). Therefore, we need not assume that the starting logic is fuzzy, and we can develop a general theory of two-layer modal logics, showing how the methods used in the fuzzy literature can lead to completeness results using very few properties of the underlying logics. As a semantics, we propose particular kinds of measured Kripke Frames and prove corresponding completeness theorems. As an illustration we will obtain Hájek's completeness result for Fuzzy Probability logic FP(Ł) over Łukasiewicz logic as a particular corollary of our general approach.

\section{The framework}

This section presents the basic definitions and notational conventions for the paper (for further information on Algebraic Logic notions see [8,4]). The definitions of a propositional language $\mathcal{L}$, the free term algebra $\boldsymbol{F} \boldsymbol{m}_{\mathcal{L}}$ over a denumerable set of generators (propositional variables), and finitary Hilbert-style proof systems are as usual. Let us introduce the notion of propositional logic that we use in this paper.

Convention 1. Let $\mathcal{L}$ be a language containing at least a truth constant $\overline{1}$ and binary connectives $\rightarrow$ and $\mathrm{V}$. In this paper a propositional logic $\mathrm{L}$ in $\mathcal{L}$ is a finitary lattice-disjunctive weakly implicative logic (as studied in [3]). In more details, this means that $\mathrm{L}$ is identified with the provability relation $\vdash_{\mathrm{L}}$ on $\boldsymbol{F m}_{\mathcal{L}}$ given by a finitary Hilbert-style system such that: ${ }^{2}$

$$
\begin{gathered}
\vdash_{\mathrm{L}} \varphi \rightarrow \varphi \quad \varphi, \varphi \rightarrow \psi \vdash_{\mathrm{L}} \psi \quad \varphi \rightarrow \psi, \psi \rightarrow \chi \vdash_{\mathrm{L}} \varphi \rightarrow \chi \quad \varphi \vdash_{\mathrm{L}} \overline{1} \rightarrow \varphi \\
\varphi \leftrightarrow \psi \vdash_{\mathrm{L}} \circ\left(\chi_{1}, \ldots \chi_{i}, \varphi, \ldots, \chi_{n}\right) \leftrightarrow \circ\left(\chi_{1}, \ldots \chi_{i}, \psi, \ldots, \chi_{n}\right) \quad \text { for every } n \text {-ary } \circ \in \mathcal{L} \text { and } i<n . \\
\vdash_{\mathrm{L}} \varphi \rightarrow \varphi \vee \psi \quad \vdash_{\mathrm{L}} \psi \rightarrow \varphi \vee \psi \quad \varphi \rightarrow \chi, \psi \rightarrow \chi \vdash_{\mathrm{L}} \varphi \vee \psi \rightarrow \chi \\
\Gamma, \varphi \vdash_{\mathrm{L}} \chi \quad \text { and } \quad \Gamma, \psi \vdash_{\mathrm{L}} \chi \quad \text { imply } \quad \Gamma, \varphi \vee \psi \vdash_{\mathrm{L}} \chi
\end{gathered}
$$

We recall now the basics of semantics. Note that our logics are algebraically implicative with a truth definition given by the single equation $x \vee \overline{1} \approx \overline{1}$. Let us fix a logic $\mathrm{L}$ in a language $\mathcal{L}$; then $\mathcal{L}$-algebras are algebras with signature $\mathcal{L}$ and homomorphisms from $\boldsymbol{F m}_{\mathcal{L}}$ to an $\mathcal{L}$-algebra $\boldsymbol{A}$ are called $\boldsymbol{A}$-evaluations. For an $\mathcal{L}$-algebra $\boldsymbol{A}$ we define the set $F_{\boldsymbol{A}}=\left\{x \mid x \vee^{A} \overline{1}^{A}=x\right\}$.

Definition 2. We say that $\boldsymbol{A}$ is an L-algebra, $\boldsymbol{A} \in \mathbb{L}$ in symbols, if

- for each $\Gamma \cup\{\varphi\} \subseteq F m_{\mathcal{L}}$ such that $\Gamma \vdash_{\mathrm{L}} \varphi$, we have that for each A-evaluation e, if $e[\Gamma] \subseteq F_{A}$, then $e(\varphi) \in F_{A}$,

- for each $x, y \in A$, if $\left\{x \rightarrow^{A} y, y \rightarrow^{A} x\right\} \subseteq F_{A}$, then $x=y$.

$\mathbb{L}$ is in fact a quasivariety and it is the equivalent algebraic semantics of $\mathrm{L}$ in the sense of [2]. A non-trivial L-algebra $\boldsymbol{A}$ is (finitely) subdirectly irreducible relative to $\mathbb{L}$ if for every (finite non-empty) subdirect representation $\alpha$ of $\boldsymbol{A}$ with a family $\left\{\boldsymbol{A}_{i} \mid i \in I\right\} \subseteq \mathbb{L}$ there is $i \in I$ such that $\pi_{i} \circ \alpha$ is an isomorphism. $\mathbb{L}_{\mathrm{R}(\mathrm{F}) \mathrm{SI}}$ denotes the class of all (finitely) subdirectly irreducible algebras relative to $\mathbb{L}$. Of course $\mathbb{L}_{\mathrm{RSI}} \subseteq \mathbb{L}_{\mathrm{RFSI}}$.

Definition 3. Let $\mathrm{L}$ be a logic and $\mathbb{K} \subseteq \mathbb{L}_{\mathrm{RFSI}}$. We say that $\mathrm{L}$ has (finite) strong $\mathbb{K}$-completeness, $\mathrm{S} \mathbb{K} \mathrm{C}$ (or FSIKC resp.) whenever for each (finite) theory $\Gamma \cup\{\varphi\}$ holds that $\Gamma \vdash_{\mathrm{L}} \varphi$ iff for each $\boldsymbol{A} \in K$ and each A-evaluation e we have $e(\varphi) \in F_{A}$ whenever $e[\Gamma] \subseteq F_{A}$.

\footnotetext{
${ }^{2}$ We write ' $\varphi \leftrightarrow \psi$ ' for ' $\{\varphi \rightarrow \psi, \psi \rightarrow \varphi\}$ ', ' $T \vdash S$ ' for ' $T \vdash \varphi$ for each $\varphi \in S$ ', and ' $T \Vdash S$ ' for ' $T \vdash S$ and $S \vdash T$ '.
} 
Algebraically we can say that L has FSKC (or S $\mathbb{K} C$ resp.) if $\mathbb{K}$ generates $\mathbb{L}$ as a $(\sigma$-)quasivariety. Note that every logic has $S L_{R S I} C$ (and hence $S L_{R F S I} C$ ). If $\mathrm{L}$ is a fuzzy logic, then $\mathbb{L}_{\mathrm{RFSI}}$ is the class of L-chains.

\section{The core theory}

Let us fix two logics $\mathrm{L}_{1}$ and $\mathrm{L}_{2}$ in disjoint languages $\mathcal{L}_{1}$ and $\mathcal{L}_{2}$ such that $\square \notin \mathcal{L}_{1} \cup \mathcal{L}_{2}$. Further we fix two classes of algebras $\mathbb{K}_{i} \subseteq\left(\mathbb{L}_{i}\right)_{\mathrm{RFSI}}, i \in\{1,2\}$. We define three kinds of formulae of a two-level language $\mathrm{Fm}_{\mathcal{L}_{2}\left(\mathcal{L}_{1}\right)}^{\mathrm{Var}}$ over the set of variables Var:

- non-modal formulae from $\mathrm{Fm}_{\mathcal{L}_{1}}^{\mathrm{Var}}$,

- atomic modal formulae of the form $\square \varphi$, for $\varphi \in F m_{\mathcal{L}_{1}}^{\mathrm{Var}}$,

- modal formulae resulting from atomic ones by connectives from $\mathcal{L}_{2}$.

Definition 4. The minimal $\mathrm{L}_{2}$-modal logic over logic $\mathrm{L}_{1}$ (denoted by $\mathrm{L}_{2}\left(\mathrm{~L}_{1}\right)$ ) has formulae $\mathrm{Fm}_{\mathcal{L}_{2}\left(\mathcal{L}_{1}\right)}^{\mathrm{Var}}$ and an axiomatic system consisting of

- the axioms and rules of $\mathrm{L}_{1}$ for non-modal formulae,

- axioms and rules of $\mathrm{L}_{2}$ for modal formulae,

- and the following congruence rule for each pair of non-modal formulae $\varphi$ and $\psi$ :

$$
\varphi \leftrightarrow \psi \vdash \square \varphi \leftrightarrow \square \psi \quad \text { (CONGR) }
$$

An $n$-ary modal rule has $n$ non-modal premises and a modal conclusion. An $\mathrm{L}_{2}$-modal logic over a logic $\mathrm{L}_{1}$ is an extension of $\mathrm{L}_{2}\left(\mathrm{~L}_{1}\right)$ by some modal rules.

We understand rules as schemata, i.e., for each substitution $\sigma$ on $F m_{\mathcal{L}_{1}}^{V a r}$, if $\varphi_{1}, \ldots, \varphi_{n} \vdash \Psi$ is a modal rule then $\sigma \varphi_{1}, \ldots, \sigma \varphi_{n} \vdash \sigma \Psi$ is also a modal rule. We define the notion of proof in a modal logic in the usual way. One can imagine that the proof consists of three separate parts: proving non-modal formulae, application of the modal rules on proved non-modal formulae, and proving modal formulae.

Definition 5. $A \mathbb{K}_{1}$-based $\mathbb{K}_{2}$-measured Kripke frame is a system $\mathbf{F}=\left\langle W,\left(\boldsymbol{A}_{w}\right)_{w \in W}, \boldsymbol{B}, \mu\right\rangle$ where $W$ is a set (of possible worlds), $\boldsymbol{A}_{w} \in \mathbb{K}_{1}$ for each $w \in W, \boldsymbol{B} \in \mathbb{K}_{2}$ and $\mu$ is a partial mapping $\mu: \prod_{w \in W} A_{w} \rightarrow B$.

Note the difference from the 'traditional' approach: in order to prove the completeness theorems in the full generality we cannot assume that all $\boldsymbol{A}_{w}$ s are the same; we call such frames uniform and we will see later in which cases we can restrict ourselves to such frames.

Definition 6. A Kripke model $\mathbf{M}$ over $\mathbb{K}_{1}$-based $\mathbb{K}_{2}$-measured Kripke frame $\mathbf{F}=\left\langle W,\left(\boldsymbol{A}_{w}\right)_{w \in W}, \boldsymbol{B}, \mu\right\rangle$ is a tuple $\mathbf{M}=\left\langle\mathbf{F},\left(e_{w}\right)_{w \in W}\right\rangle$ where:

- $e_{w}: F_{\mathcal{L}_{1}}^{V a r} \rightarrow A_{w}$ is an $\boldsymbol{A}_{w}$-evaluation,

- for each non-modal formula $\varphi$, the element $\varphi_{\mathbf{M}} \in \prod_{w \in W} A_{w}$ defined as $\varphi_{\mathbf{M}}(w)=e_{w}(\varphi)$ belongs to the domain of $\mu$.

The truth value of atomic modal formulae is defined (uniformly for all worlds) as:

$$
\|\square \varphi\|_{\mathbf{M}}=\mu\left(\varphi_{\mathbf{M}}\right) ;
$$

and the truth value of non-atomic modal formulae is (uniformly) computed by using operations from $\boldsymbol{B}$. 
We say that $\mathbf{M}$ is a satisfies the (non-)modal formula $\Psi$ ( $\psi$ resp.) whenever $\|\Psi\|_{\mathbf{M}} \in F_{\boldsymbol{B}}$ (or $e_{w}[\psi] \in$ $F_{\boldsymbol{A}_{w}}$ for each $w \in W$ respectively).

Finally we say that $\mathbf{F}$ is a frame for an $\mathrm{L}_{2}$-modal logic over a logic $\mathrm{L}_{1}$ if all its additional modal rules are valid in all Kripke models over $\mathbf{F}$, i.e. the conclusion of a modal rule is satisfied in all models of over $\mathbf{F}$ which satisfy all its premises.

Next we state the main theorem, the completeness of an $\mathrm{L}_{2}$-modal logic over a logic $\mathrm{L}_{1}$. We will see that the form/strength of the completeness we obtain depends on the form/strength of the completeness of the logics $\mathrm{L}_{2}$ and $\mathrm{L}_{1}$. The proof has two main ingredients: Hájek's idea from [11] of a translation of formulae and proofs from an $\mathrm{L}_{2}$-modal logic over a $\operatorname{logic} \mathrm{L}_{1}$ into the $\operatorname{logic} \mathrm{L}_{2}$ and the authors' characterization of completeness properties from [3].

Theorem 7. Let $\mathrm{L}$ be an $\mathrm{L}_{2}$-modal logic over a logic $\mathrm{L}_{1}$ such that $\mathrm{L}_{i}$ has $\mathrm{S}_{i} \mathrm{C}$. Then the following are equivalent for each non-modal theory $T$, modal theory $\Gamma$, and a modal formula $\Phi$ :

- $\Gamma, T \vdash_{\mathrm{L}} \Phi$

- for each $\mathbb{K}_{1}$-based $\mathbb{K}_{2}$-measured Kripke frame $\mathbf{F}$ for L and each Kripke model $\mathbf{M}$ over $\mathbf{F}$ holds that $\mathbf{M}$ satisfies $\Phi$ whenever it satisfies all formulae from $\Gamma$ and $T$.

The same equivalence holds if $\mathrm{L}_{2}$ has $\mathrm{FS} \mathbb{K}_{2} \mathrm{C}$ only but at the price of restricting to finite $\Gamma$ and $T$ and additional assumptions that $\mathbb{L}_{1}$ is a locally finite and $\mathrm{L}$ has only finitely many additional modal rules.

Note that any $\mathrm{L}_{2}$-modal logic over a logic $\mathrm{L}_{1}$ enjoys completeness w.r.t. its $\left(\mathbb{L}_{1}\right)_{\mathrm{RFSI}}$-based $\left(\mathbb{L}_{2}\right)_{\mathrm{RFSI}}{ }^{-}$ measured Kripke frames and if $\mathrm{L}_{1}$ enjoys completeness w.r.t. a single algebra, then we can restrict ourselves to uniform frames/models.

\section{A case study: fuzzy probability logic over Boolean logic}

As a case study we repeat the definition of Fuzzy Probability logic over Eukasiewicz logic Ł [13, 11], denoted as FP(Ł) for short, and show how its completeness follows from our main theorem. Recall that $\mathrm{FP}(\mathrm{E})$ is given by the following axiomatic system:

- axioms of classical propositional logic Bool for non-modal formulae and axioms $€$ for modal ones,

- modus ponens rules for both non-modal and modal formulae,

- axioms $\square \varphi \rightarrow(\square(\varphi \rightarrow \psi) \rightarrow \square \psi), \square(\neg \varphi) \leftrightarrow \neg \square(\varphi)$, and $\square(\varphi \vee \psi) \leftrightarrow[(\square \varphi \rightarrow \square(\varphi \wedge \psi)) \rightarrow \square \psi]$,

- modal rule $\varphi \vdash \square \varphi$.

Clearly the logic FP( $($ ) is an Ł-modal logic over Bool with only finitely many additional modal rules; Bool is locally finite and enjoys S2C; and $€$ enjoys FS[0,1 $]_{€} \mathrm{C}$. Thus we can use our main theorem. In order to obtain exactly Hájek's formulation we also observe that $\mathbf{F}=\left\langle W,\left(\boldsymbol{A}_{w}\right)_{w \in W}, \boldsymbol{B}, \mu\right\rangle$ is a $\{\mathbf{2}\}$-based $\left\{[0,1]_{\mathrm{E}}\right\}$-measured Kripke frame for $\mathrm{FP}(\mathrm{E})$ iff $\mu$ is finitely additive probability measure.

Theorem 8 ([11, Theorem 8.1.14]). Let $T$ and $\Gamma$ be finite non-modal and modal (resp.) theories in $\mathrm{FP}(\mathrm{\leftarrow})$ and let $\Phi$ be a modal formula. Then the following are equivalent:

- $\Gamma, T \vdash_{\mathrm{FP}(€)} \Phi$

- $\|\Phi\|_{\mathbf{M}}=1$ for all Kripke models $\mathbf{M}$, satisfying $\Gamma$ and $T$, over all $\{2\}$-based $[0,1]_{\mathrm{E}}$-measured Kripke frames, where $\mu$ is a finitely additive probability measure. 


\section{References}

[1] L. Běhounek, P. Cintula, and P. Hájek. Introduction to mathematical fuzzy logic. In P. Cintula, P. Hájek, and C. Noguera, editors, Handbook of Mathematical Fuzzy Logic - Volume 1, volume 37 of Studies in Logic, Mathematical Logic and Foundations, pages 1-101. College Publications, London, 2011.

[2] W. J. Blok and D. L. Pigozzi. Algebraizable Logics, volume 396 of Memoirs of the American Mathematical Society. American Mathematical Society, Providence, RI, 1989. Freely downloadable from http://orion. math.iastate.edu/dpigozzi/

[3] P. Cintula and C. Noguera. A general framework for mathematical fuzzy logic. In P. Cintula, P. Hájek, and C. Noguera, editors, Handbook of Mathematical Fuzzy Logic - Volume 1, volume 37 of Studies in Logic, Mathematical Logic and Foundations, pages 103-207. College Publications, London, 2011.

[4] J. Czelakowski. Protoalgebraic Logics, volume 10 of Trends in Logic. Kluwer, Dordrecht, 2001.

[5] T. Flaminio and L. Godo. A logic for reasoning about the probability of fuzzy events. Fuzzy Sets and Systems, 158(6):625-638, 2006.

[6] T. Flaminio, L. Godo, and E. Marchioni. Reasoning about uncertainty of fuzzy events: An overview. In P. Cintula, C. Fermüller, and L. Godo, editors, Understanding Vagueness: Logical, Philosophical, and Linguistic Perspectives, volume 36 of Studies in Logic, pages 367-400. College Publications, London, 2011.

[7] T. Flaminio and F. Montagna. A logical and algebraic treatment of conditional probability. Archive for Mathematical Logic, 44(2):245-262, 2005.

[8] J. M. Font, R. Jansana, and D. L. Pigozzi. A survey of Abstract Algebraic Logic. Studia Logica, 74(1-2, Special Issue on Abstract Algebraic Logic II):13-97, 2003.

[9] L. Godo, F. Esteva, and P. Hájek. Reasoning about probability using fuzzy logic. Neural Network World, 10(5):811-823, 2000. Special issue on SOFSEM 2000.

[10] L. Godo and E. Marchioni. Coherent conditional probability in a fuzzy logic setting. Logic Journal of the Interest Group of Pure and Applied Logic, 14(3):457-481, 2006.

[11] P. Hájek. Metamathematics of Fuzzy Logic, volume 4 of Trends in Logic. Kluwer, Dordrecht, 1998.

[12] P. Hájek and P. Cintula. On theories and models in fuzzy predicate logics. Journal of Symbolic Logic, 71(3):863-880, 2006.

[13] P. Hájek, L. Godo, and F. Esteva. Fuzzy logic and probability. In Proceedings of the 11th Annual Conference on Uncertainty in Artificial Intelligence UAI '95, pages 237-244, Montreal, 1995.

[14] E. Marchioni. Possibilistic conditioning framed in fuzzy logics. International Journal of Approximate Reasoning, 43(2):133-165, 2006.

[15] P. Rusnok. Probability in formal fuzzy logic. Master thesis, Czech Technical University in Prague, 2008. 